\title{
Cosmetic Applications of Regenerative Medicine-A New Approach to the Reduction of Neck Lines
}

\author{
E. Russell Vickers, Haitao Wen \\ Clinical Stem Cells Pty Ltd., Sydney, Australia \\ Email: manager@clinicalstemcells.com
}

How to cite this paper: Vickers, E.R. and Wen, H.T. (2020) Cosmetic Applications of Regenerative Medicine-A New Approach to the Reduction of Neck Lines. Open Journal of Regenerative Medicine, 9, 36-42. https://doi.org/10.4236/ojrm.2020.92005

Received: April 1, 2020

Accepted: April 17, 2020

Published: April 20, 2020

Copyright ( 2020 by author(s) and Scientific Research Publishing Inc. This work is licensed under the Creative Commons Attribution International License (CC BY 4.0).

http://creativecommons.org/licenses/by/4.0/

\section{(c) (i) Open Access}

\begin{abstract}
Combining different areas of regenerative medicine is considered the new standard for comprehensive tissue and organ regeneration. This approach can be further applied to cosmetic issues such as deep neck lines. This case report utilized techniques of autologous stem cell therapy and epigenetic tissue environment modulation with externally applied polyphenols and tissue penetration agents. The results showed a remarkable cosmetic improvement with a significant reduction of neck lines in a patient where employment and psychological issues were developing from the original problem. The regenerative medicine protocol was safe, efficient in disposable materials, portable benchtop equipment and surgical operating time. The patient had an excellent outcome for this difficult to treat cosmetic problem.
\end{abstract}

\section{Keywords}

Regenerative Medicine, Cosmetic Medicine, Autologous Stem Cells, Polyphenols, Psychology

\section{Introduction}

Regenerative medicine is a rapidly developing of medicine incorporating diverse but integrated fields of stem cells, bioscaffolds, biomarkers, molecular epigenetics and DNA editing. Substantial research on regenerative medicine has focused on replacing diseased tissues and organs such as heart, liver, kidneys and pancreas [1]. The use of regenerative medicine technology for treating cosmetic problems is gaining popularity in many parts of Asia and western countries. China, Japan and South Korea have large percentages of the general population with disposable incomes for spending on high quality health 
treatments and cosmetic services. Current cosmetic treatments of the face and head region include surgical facelifts, hair transplantation, cheek and chin augmentation, removal of wrinkles and aging lines, injections of fillers and botulinum.

Other applications show a crossover of medical and cosmetic medicine such as burns victims and with difficult decision-making placed on immediate relatives [2]. The regenerative approach to treat the skin of burn victims is most important for improved outcomes on both the psychological and physical functional dimensions of afflicted patients. Developing in parallel to these treatments is the cosmetic field, particularly on skin regeneration to obtain a more youthful appearance. Facial and neck aesthetics have major importance in a large percentage of the population, especially women, but with an increasing awareness and utilization of cosmetic products by men. This can be observed in the time taken by people to apply makeup and style hair. Looking young has important positive psychological benefits that translate into better physical and emotional health, and better employment prospects in many industries. In contrast, there can be significant psychological comorbidities in people with facial conditions requesting regenerative treatments [3].

Facial wrinkles, deepening neck lines and hair loss are obvious cosmetic markers of ageing. The cause can be genetic conditions such as progeria or more frequent ultraviolet damage to skin causing loss of elastin and collagen resulting in wrinkles and skin cancer [4]. The cosmetic market for face and neck improvement is measured in tens of billions of dollars each year in the world. More medical doctors are focusing their attention specifically to cosmetic medicine, and the public now commits significant time and money to investigate and use cosmetic medicine.

Current treatment for removal of neck lines includes plastic surgery but this can cause undesirable effects such as a stretched skin appearance and leave visible scar lines at the surgical incision sites. This can result in a psychologically devastated patient. Moreover, further surgery is more difficult due to loss of available tissue volume and increased scar tissue formation. Cosmetic outcomes from failed plastic surgery can be irreversible. Other treatments include cosmetic fillers, thermofrequency and lasers on deep neck and face lines that give temporary benefit, are painful, or require multiple attempts.

This case report demonstrates the remarkable outcome of utilizing regenerative medicine for the treatment of multiple neck lines. Our protocol combined autologous adipose tissue derived stem cells with a new scientifically designed topical serum to augment the stem cell differentiation. The serum was formulated to be a highly absorbent topical serum for skin and was applied several times each day after the stem cell injections. The purpose of this serum was for the bioactive compounds to penetrate skin and promote the stem cells to fibroblasts for expression of collagen and elastin to give a more permanent and natural looking effect. 


\section{Case Presentation}

A 37-year-old Chinese female in good health sought treatment for extensive and multiple neck lines. Her occupation was a professional singer and the appearance of neck lines was affecting her psychologically and employment prospects. Photos of the neck only were taken to maintain anonymity and were recorded at baseline, and postoperatively at 2 days, 5 days and 5 weeks. The subject gave permission for the images to be used for research publication purposes.

The stem cell therapy used a variation of the technique previously published by the author using stem cells for facial pain and inflammation [5]. In addition, a cosmetic biochemistry approach was included. This consisted of daily self administered applications of a highly absorbable topical serum with a novel formulation of bioactive compounds with stem cell signaling properties.

Tumescent liposuction was used to acquire autologous adipose mesenchymal stem cells. Preoperatively the subject was given $1 \mathrm{gm}$ oral systemic cephalosporin antibiotics. The subject was draped and skin antiseptic applied to the abdominal region. $10 \mathrm{ml}$ of local anesthetic (2\% lignocaine with 1:100,000 adrenalin) was mixed with $10 \mathrm{ml} 0.9 \%$ normal saline. A small scalpel incision was performed through the dermis and a $10 \mathrm{~cm} 14$ gauge liposuction cannula with a blunt end and internal cutting edge was inserted. Manual suction was accomplished with a $50 \mathrm{ml}$ syringe and a plunger lock mechanism to maintain vacuum. $7 \mathrm{gm}$ of tumescent lipoaspirate was obtained and then subjected to manual passaging to emulsify the fat. The emulsified product was vortexed at high speed for $4 \mathrm{~min}$ and then centrifuged for $10 \mathrm{~min}$ at $4000 \mathrm{rpm}$ at room temperature $20^{\circ} \mathrm{C}$. The cellular stromal vascular fraction (SVF) was removed and reconstituted in $10 \mathrm{ml}$ of normal saline. Surgery was performed by author ERV (maxillofacial surgeon) and technical procedures performed by author HW.

The neck lines were injected with local anaesthetic in small $0.1 \mathrm{ml}$ volume increments. The SVF containing stem cells was injected intradermally along the neck lines with a 25 gauge needle. The subject was given the topical serum formulation and instructed to apply to the neck lines twice daily for 4 weeks.

The subject reported no adverse effects or complications from the procedure. There was minor visible redness and swelling of the neck lines at the injections sites for 2 days. There was minimal discomfort and no analgesics were taken by the subject. There was rapid and visible improvement of the necklines at 5 days and the reduction of neck lines continued for 5 weeks. The subject was very satisfied with the result (Figures 1-4).

\section{Discussion}

Consensus among scientists is the using of multimodal regenerative techniques applicable to the target tissue or organ. Specific for this case of skin neck lines was the administration of stem cells directly to the target site to precisely locate the stem cells. This is a straightforward and simple medical approach using 23 25 gauge needle. The surgical protocol was adapted from our previous work to 


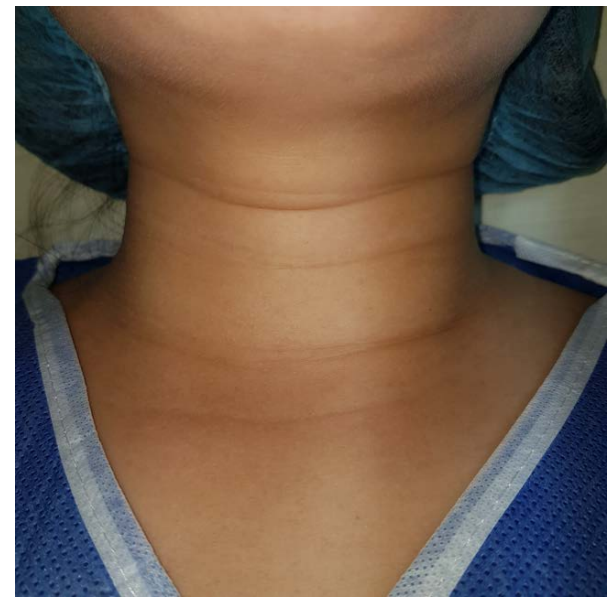

Figure 1. 39-year-old female before treatment. Image shows a total of 3 major deep neck lines, and 8 minor branched neck lines.

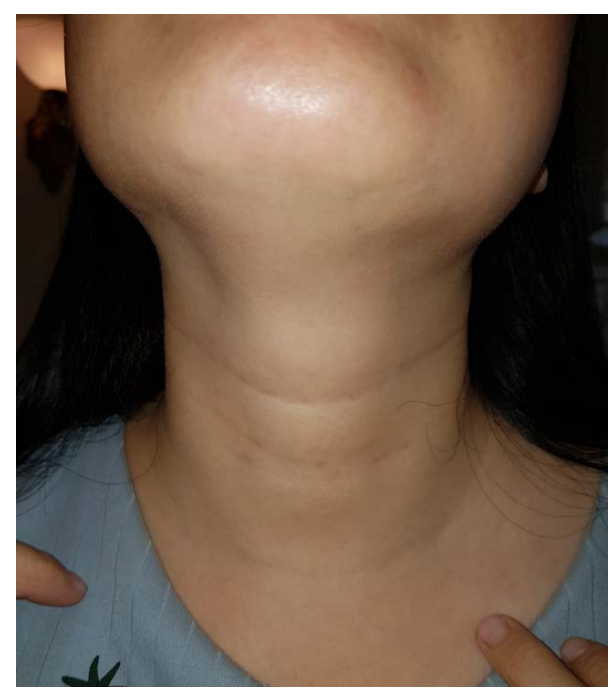

Figure 2. 2 days after regenerative medicine treatment. Minor redness from the injections present and diminishing of minor neck lines. The patient reported no pain and did not require any analgesics.

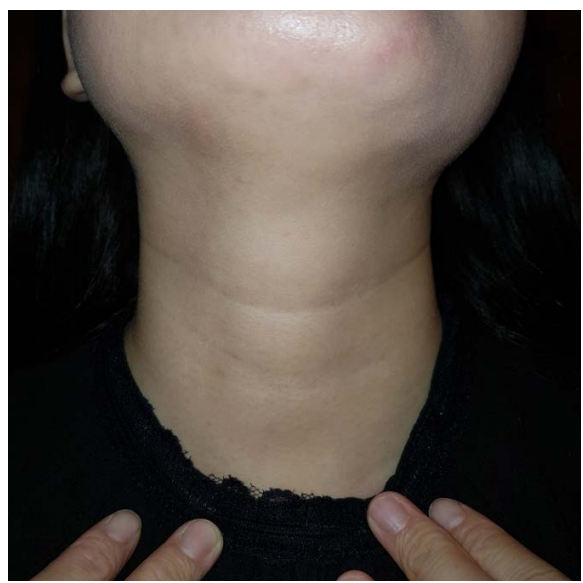

Figure 3. 5 days after injections. The minor lines have further reduced and the major lines are reducing. 


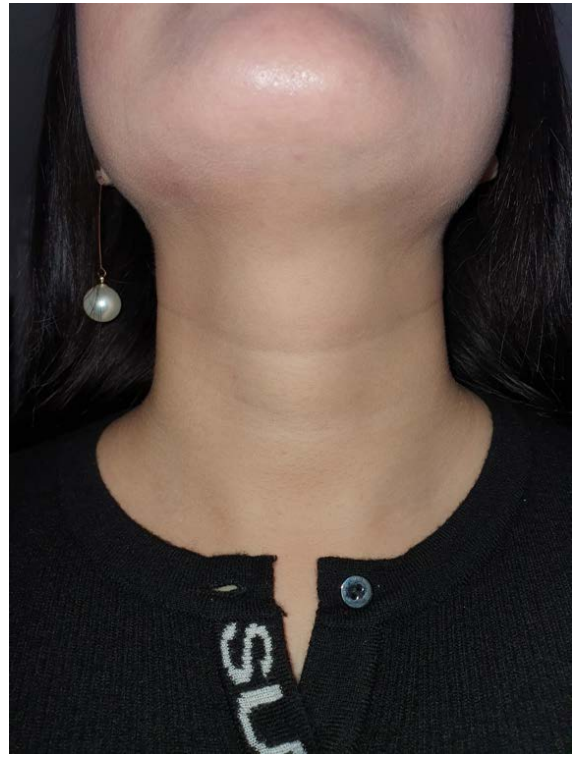

(a)

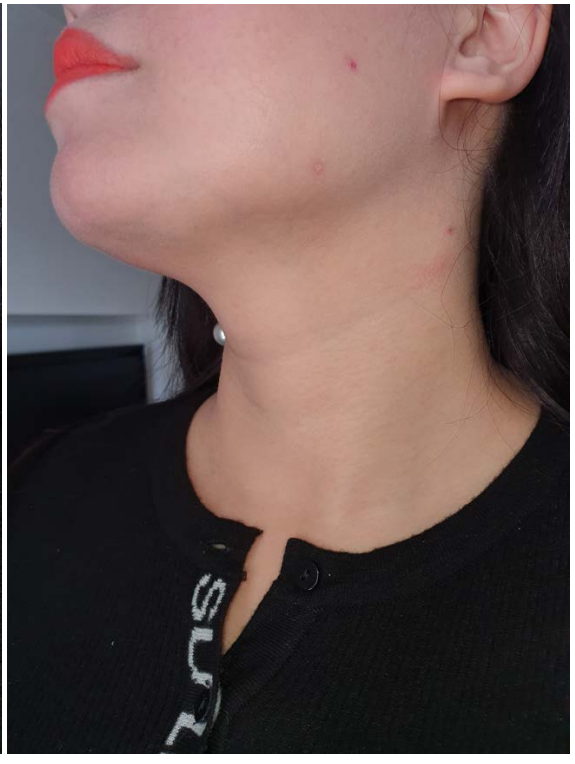

(b)

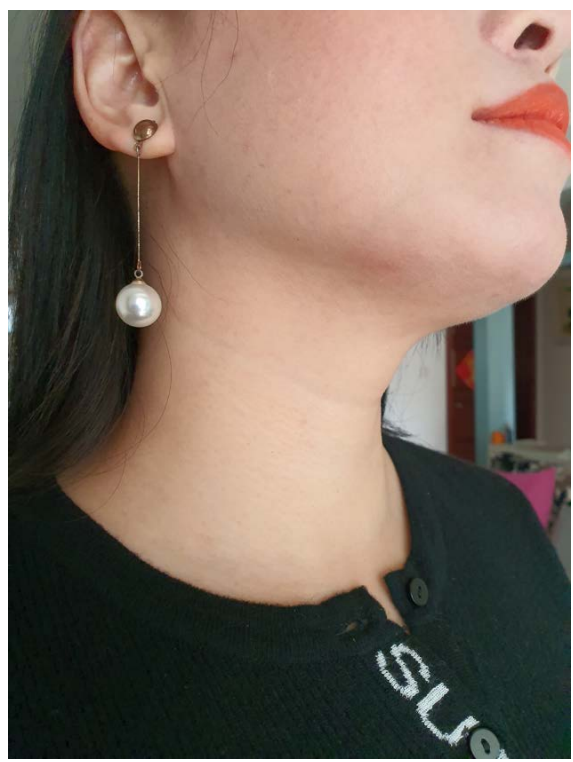

(c)

Figure 4. 5 weeks after injections and using the skin serum. Frontal (a) and lateral (b, c) images show minor neck lines have disappeared. There has been a significant improvement in the 3 major neck lines. (a) Frontal image of neck at 5 weeks after treatment; (b) Lateral image of the left neck at 5 weeks after treatment; (c) Lateral image of the right neck at 5 weeks after treatment.

increase efficiency and safety of the technique. All surgical components and materials were sterile and disposable, preventing cross contamination and post operative infection with micro-organisms such as antibiotic resistant staphylococcus found on the face. The SVF is performed under a closed loop system to prevent infection. This was followed by use of small benchtop vortex mixer and centrifuge. The laboratory equipment is standardized and portable. No expensive equipment such as a biosafety cabinet was required. In addition, there was a 
significant cost reduction as no collagenase was used for enzymatic digest of the fat.

The topical cream was specifically formulated with known bioactive compounds to enhance cell metabolism and stem cell differentiation. Most of these compounds have shown in vitro and ex vivo effects in the published literature. Moreover, the compounds are very safe and are found in foods, particularly herbs such as green tea and its polyphenol key active of epigallocatechin gallate. Many polyphenols are lipophilic and require solvents, emulsifiers and surfactants that are suitable for oil in water topical creams. We have achieved this approach using novel natural solvents based on molecular cell membrane lipid transport mechanisms. This achieved excellent solubility of lipid polyphenols in the serum without having to use synthetic chemicals that may cause local inflammation, thereby negating stem cell signaling to the local niche of skin resident stem cells. We are further enhancing this serum for wider applications in cosmetic and regenerative medicine.

The pathogenesis of wrinkles is complex. At the microstructural level there is depletion of elastin and collagen that macroscopically and aesthetically results in wrinkles and sagging of tissue. In addition, dysregulation of lymphatic vessels and surrounding perilymphatic fat in the dermis leads to inflammation, scarring, and fibrosis [6]. Biochemically, matrix metalloproteinases (MMPs - collagenase enzymes) degrade collagen. Previous research (photoaged rat model) has shown injections of cultured fibroblasts increase collagen but also increase MMPs. Injections of adipose stem cells, however, increased collagen and decreased MMPs [7] suggesting that stem cells are the preferred cell type for clinical use. Moreover, adipose stem cells have a repertoire of anti-inflammatory cytokines in their secretome increasing the immunomodulatory paracrine effect of the dysregulated cellular matrix in wrinkles [8].

\section{Conclusions}

This case report demonstrates the approach of integrating different areas of the regenerative medicine field to achieve a significant improvement on skin aesthetics [9]. The average cost of a neck lift is $\$ 5242$, according to 2018 statistics from the American Society of Plastic Surgeons [10] and involves general anaesthesia and significant bruising and swelling of the neck for several weeks. It is obvious to the patient's personal friends and can create unnecessary negative comments. Similar issues exist for a surgical face lift (\$7655) [10]. Furthermore, in conventional surgery of the neck and face, there are the potential major implications and complications of paresis and paraesthesia to the cosmetic patient due to the superficial proximity of motor branches of the facial nerve and sensory divisions of the trigeminal nerve [11].

In contrast, our described surgical protocol was simple, safe and effective. Importantly, the new technique maintained the patient's privacy of having a cosmetic procedure. Postoperatively, it was not obvious to other work related 
people and friends. The topical serum formulation was developed by using a novel combination of bioactive compounds with enhanced skin penetration chemistry. The described regenerative medicine methodology is applicable for further applications of cosmetic medicine.

\section{Conflicts of Interest}

The authors declare no conflicts of interest regarding the publication of this paper.

\section{References}

[1] Madeddu, P., Avolio, E., Alvino, V.V., Santopaolo, M. and Spinetti, G. (2019) Personalized Cardiovascular Regenerative Medicine: Targeting the Extreme Stages of Life. Frontiers in Cardiovascular Medicine, 6, 177. https://doi.org/10.3389/fcvm.2019.00177

[2] Liu, Y.M. and Romanowski, K.S. (2018) Should Cosmetic Outcome Influence Discussions about Goals of Care for Severely Burned Patients? AMA Journal of Ethics, 20, 546-551. https://doi.org/10.1001/journalofethics.2018.20.6.cscm3-1806

[3] Braunsteiner, N.E., Vickers, E.R. and Shparberg, R. (2018) Psychological Issues for Patients Undergoing Stem Cell Therapy and Regenerative Medicine. Open Journal of Regenerative Medicine, 7, 1-17. https://doi.org/10.4236/ojrm.2018.71001

[4] Narayanan, D.L, Saladi, R.N. and Fox, J.L. (2010) Ultraviolet Radiation and Skin Cancer. International Journal of Dermatology, 49, 978-986.

https://onlinelibrary.wiley.com/doi/pdf/10.1111/j.1365-4632.2010.04474.x https://doi.org/10.1111/j.1365-4632.2010.04474.x

[5] Vickers, E.R., Karsten, E., Flood, J. and Lilischkis, R. (2014) A Preliminary Report on Stem Cell Therapy for Neuropathic Pain in Humans. Journal of Pain Research, 8, 255-263. https://doi.org/10.2147/JPR.S63361

[6] Pessa, J.E., Nguyen, H., John, G.B. and Scherer, P.E. (2014) The Anatomical Basis for Wrinkles. Aesthetic Surgery Journal, 34, 227-234.

https://doi.org/10.1177/1090820X13517896

[7] Jeong, J.H., Fan, Y., You, G.Y., Choi, T.H. and Kim, S. (2015) Improvement of Photoaged Skin Wrinkles with Cultured Human Fibroblasts and Adipose-Derived Stem Cells: A Comparative Study. Journal of Plastic and Reconstructive Aesthetic Surgery, 68, 372-381. https://doi.org/10.1016/j.bjps.2014.10.045

[8] Guillén, M.I., Platas, J., Pérez Del Caz, M.D., Mirabet, V. and Alcaraz, M.J. (2018) Paracrine Anti-Inflammatory Effects of Adipose Tissue-Derived Mesenchymal Stem Cells in Human Monocytes. Frontiers in Physiology, 9, 661. https://doi.org/10.3389/fphys.2018.00661

[9] Vickers, E.R. (2016) Stem Cells and Cosmetic Science. Journal of the Australian Society of Cosmetic Chemists, 6, 29-42. https://ae4e433d-5255-4d24-9465-764b558439c1.filesusr.com/ugd/46ff6c_4a779b56 96b74f16a5d1d52e4843a9f4.pdf

[10] https://www.plasticsurgery.org/cosmetic-procedures/neck-lift/cost

[11] Chaffoo, R.A.K. (2013) Complications in Facelift Surgery: Avoidance and Management. Facial Plastic Surgery Clinics North America, 21, 551-558.

https://doi.org/10.1016/j.fsc.2013.07.007 